\title{
A THEORETICAL DERIVATION OF THE CONDENSED HISTORY ALGORITHM
}

\author{
EDWARD W. LARSEN \\ Department of Nuclear Engineering \\ University of Michigan \\ Ann Arbor, Michigan 48109 USA
}

To Professor Jacques Devooght on his sixtieth birthday.

\begin{abstract}
Although the Condensed History Algorithm is a successful and widely-used Monte Carlo method for solving electron transport problems, it has been derived only by an ad-hoc process based on physical reasoning. In this paper we show that the Condensed History Algorithm can be justified as a Monte Carlo simulation of an operator-split procedure in which the streaming, angular scattering, and slowing-down operators are separated within each time step. Different versions of the operator-split procedure lead to $O(\Delta s)$ and $O\left(\Delta s^{2}\right)$ versions of the method, where $\Delta s$ is the path-length step. Our derivation also indicates that higher-order versions of the Condensed History Algorithm may be developed.
\end{abstract}

\section{INTRODUCTION}

Analog Monte Carlo simulations of particle transport processes are a direct imitation of physical reality. In such simulations, Monte Carlo particles are born; they travel a certain distance (determined by a probability distribution function) to the site of a collision; they scatter or are absorbed (according to certain known probabilities); and if they scatter, they emerge from the collision with a new direction of flight and energy (determined again by probability distribution functions). This process, repeatedly applied to each Monte Carlo particle until its absorption or leakage from the system, directly imitates the individual history of a physical particle. A basic difference between analog Monte Carlo and physical reality is that analog Monte Carlo generally uses many fewer particles than an actual physical problem. This often causes significant statistical errors; these can sometimes be reduced by employing non-analog or "variance reduction" techniques.

A second difficulty occurs with Monte Carlo simulations of electron transport problems. In the process of slowing down within a system, typical fast electrons undergo about $10^{5}$ collisions, for most of which the electrons' directions and energies are only slightly changed. Because of this large number of interactions, analog simulations of electron histories require an inordinate computational effort. The Condensed History Algorithm ${ }^{1-3}$ has been developed to cope with this difficulty. In this method, the physical electron transport process is approximated, rather than directly imitated. The essence of the approximation is that: $(i)$ Monte Carlo electrons do not undergo collisions at 
the rapid rate dictated by physics, but instead at a much slower rate dictated by the code user, and (ii) each Monte Carlo or "macro"-collision approximately describes the cumulative effect of a large number of physical collisions. The Condensed History Algorithm uses the fact that individual collisions between electrons and atoms generally have an extremely small effect on an electron's energy and direction. Thus, the accumulation of many physical collisions in a single macro-collision also generally has a small effect on an electron's energy and direction.

Comparisons with physical experiments and analog Monte Carlo calculations have shown that the Condensed History Algorithm yields accurate results for many types of problems. However, previous derivations of the method have been based on physical reasoning. These derivations do not provide a sound theoretical foundation for the method, nor do they provide rigorous ways of improving it. In this paper we give a mathematical justification of the Condensed History Algorithm as a Monte Carlo simulation of an operator-split procedure. We show that for conventional versions of the method, the truncation error due to the operator split is $O(\Delta s)$, where $\Delta s$ is the path-length step. However, we derive a second-order $O\left(\Delta s^{2}\right)$ version of the method, and we also discuss the derivation of higher-order versions. The basic purpose of this paper is theoretical in nature; concerns such as numerical testing and comparisons are left for future work.

The remainder of this paper is organized as follows. In Sec. II we give a physical derivation of the Condensed History Algorithm along the lines outlined by Berger. ${ }^{3}$ In Sec. III we calculate the order of the truncation errors for various versions of the method. We conclude with a brief discussion in Sec. IV.

\section{PHYSICAL DERIVATION}

In this section we shall describe the Condensed History Algorithm as a Monte Carlo simulation of the transport equation with the continuous slowing down approximation:

$$
\begin{aligned}
& \frac{1}{v} \frac{\partial \psi}{\partial t}(\underline{r}, \underline{\Omega}, E, t)+\underline{\Omega} \cdot \underline{\nabla} \psi(\underline{r}, \underline{\Omega}, E, t)+\sigma_{s}(E) \psi(\underline{r}, \underline{\Omega}, E, t) \\
& \quad=\int \sigma_{s}\left(\underline{\Omega} \cdot \underline{\Omega}^{\prime}, E\right) \psi\left(\underline{r}, \underline{\Omega^{\prime}}, E, t\right) d \Omega^{\prime}+\frac{\partial}{\partial E} \beta(E) \psi(\underline{r}, \underline{\Omega}, E, t) .
\end{aligned}
$$

Here $\underline{r}$ denotes position, $\underline{\Omega}$ is a unit vector denoting the direction of electron flight, $E$ is energy, and $t$ is time. Also, $\sigma_{s}\left(\underline{\Omega} \cdot \underline{\Omega}^{\prime}, E\right)$ is the differential scattering cross section,

$$
\sigma_{s}(E)=\int \sigma_{s}\left(\underline{\Omega} \cdot \underline{\Omega^{\prime}}, E\right) d \Omega
$$

is the macroscopic scattering cross section, $\beta(E)$ is the stopping power, $v$ is the electron speed, and

$$
\begin{gathered}
\psi(r, \underline{\Omega}, E, t) d^{3} r d \Omega d E=\text { the probable number of electrons in } d^{3} r \text { about } \\
\underline{r}, \text { in } d \Omega \text { about } \underline{\Omega}, \text { and in } d E \text { about } E, \text { at time } t .
\end{gathered}
$$

We assume that the initial electron population for $t=0$ is prescribed:

$$
\psi(\underline{r}, \underline{\Omega}, E, 0)=\psi^{i}(\underline{r}, \underline{\Omega}, E) \quad .
$$

In this paper we shall consider Eq. (1) as holding within an infinite homogeneous domain and ignore the presence of boundaries or interfaces. The physical model described by Eq. (1) includes 
(i) elastic interactions between electrons and atoms in which the direction of electron flight changes but the electron's energy is assumed to be unchanged, and (ii) inelastic small-energy-loss interactions, modeled by a stopping power, in which the electron's direction is assumed to be unchanged. Electrons can also undergo rare "catastrophic" inelastic collisions in which they lose a significant fraction of their energy, but for simplicity we shall not include such terms in this paper.

The Condensed History Algorithm artificially decouples the processes of streaming, angular scattering, and slowing down in Eq. (1). Thus, we shall now discuss each of these processes separately.

First, the transport equation that describes streaming is

$$
\frac{\partial \psi}{\partial s}(\underline{r}, \underline{\Omega}, E, s)+\underline{\Omega} \cdot \underline{\nabla} \psi(\underline{r}, \underline{\Omega}, E, s)=0
$$

where $s=v t$ is the path length tracked by an electron with speed $v$. (We shall say that Eq. (2) describes "transport process A.") Eq. (2) can be simulated by a "deterministic" or non-random Monte Carlo procedure. Specifically, at $s=s_{n}$, let a particle have position $\underline{r}_{n}$, direction $\underline{\Omega}_{n}$, and energy $E_{n}$. Then, Eq. (2) dictates that at $s=s_{n+1}$, the particle's position, direction, and energy are deterministically given by

$$
\begin{aligned}
\underline{r}_{n+1} & =\underline{r}_{n}+\underline{\Omega}_{n} \Delta s_{n}, \\
\underline{\Omega}_{n+1} & =\underline{\Omega}_{n}, \\
E_{n+1} & =E_{n},
\end{aligned}
$$

where

$$
\Delta s_{n}=s_{n+1}-s_{n}
$$

Since process A does not include angular scattering or slowing down, the direction and energy of each particle is unchanged.

Next, let us consider the transport process that includes angular scattering but does not include space-dependence or slowing down. This is described by Eq. (1) with $0=\underline{\Omega} \cdot \underline{\nabla} \psi=\beta(E)$ :

$$
\frac{\partial \psi}{\partial s}(\underline{r}, \underline{\Omega}, E, s)+\sigma_{s}(E) \psi(\underline{r}, \underline{\Omega}, E, s)=\int \sigma_{s}\left(\underline{\Omega} \cdot \underline{\Omega^{\prime}}, E\right) \psi\left(\underline{r}, \underline{\Omega^{\prime}}, E, s\right) d \Omega^{\prime}
$$

(We shall say that this describes "transport process B.") Eq. (3) can be simulated by a straightforward Monte Carlo process. As before, let us suppose that at $s=s_{n}$, a particle has position $\underline{r}_{n}$, direction $\underline{\Omega}_{n}$, and energy $E_{n}$. Let us choose coordinate vectors $\underline{i}, \underline{j}$, and $\underline{k}$ so that $\underline{k}=\underline{\Omega}_{n}$ and

$$
\underline{\Omega}=\sqrt{1-\mu^{2}}(\cos \phi \underline{i}+\sin \phi \underline{j})+\mu \underline{k},
$$

where $\mu$ is the cosine of the polar angle and $\phi$ is the azimuthal angle. Then, integrating Eq. (3) over $\phi$, we obtain

$$
\frac{\partial \hat{\psi}}{\partial s}(\underline{r}, \mu, E, s)+\sigma_{s}(E) \hat{\psi}(\underline{r}, \mu, E, s)=\int_{-1}^{1} \hat{\sigma}_{s}\left(\mu, \mu^{\prime}\right) \hat{\psi}\left(\underline{r}, \mu^{\prime}, E, s\right) d \mu^{\prime}
$$

where

$$
\hat{\psi}(\underline{r}, \mu, E, s)=\int_{0}^{2 \pi} \psi(\underline{r}, \underline{\Omega}, E, s) d \phi
$$




$$
\hat{\sigma}_{s}\left(\mu, \mu^{\prime}\right)=\int_{0}^{2 \pi} \sigma_{s}\left(\underline{\Omega} \cdot \underline{\Omega^{\prime}}, E\right) d \phi=\sum_{m=0}^{\infty} \frac{2 m+1}{2} \sigma_{s m}(E) P_{m}(\mu) P_{m}\left(\mu^{\prime}\right)
$$

and

$$
\sigma_{s 0}(E)=\sigma_{s}(E)
$$

Here, $P_{m}(\mu)$ are Legendre polynomials and $\sigma_{s m}(E)$ are known functions; Eq. (5) follows from the well-known addition theorem for spherical harmonics. ${ }^{4}$

The solution of Eq. (4) with the "initial" condition

$$
\hat{\psi}\left(\underline{r}, \mu, E, s_{n}\right)=\psi_{n} \delta\left(r-r_{n}\right) \delta\left(E-E_{n}\right) \delta(\mu-1)
$$

is easily found to be

$$
\hat{\psi}(\underline{r}, \mu, E, s)=\psi_{n} \delta\left(\underline{r}-\underline{r}_{n}\right) \delta\left(E-E_{n}\right) \psi^{*}(\mu, E, s) \quad, \quad s \geq s_{n}
$$

where

$$
\psi^{*}(\mu, E, s)=\sum_{m=0}^{\infty} \frac{2 m+1}{2} P_{m}(\mu) e^{-\left[\sigma_{s 0}(E)-\sigma_{s m}(E)\right]\left(s-s_{n}\right)} \quad, \quad s \geq s_{n} .
$$

This is the well-known Goudsmit-Saunderson distribution. ${ }^{5}$ Since

$$
\int_{-1}^{1} \psi^{*}(\mu, E, s) d \mu=1 \quad, \quad s \geq s_{n}
$$

then for any $s=s_{n+1}>s_{n}$, we have the following interpretation:

$$
\begin{gathered}
\psi^{*}\left(\mu, E, s_{n+1}\right) d \mu=\left\{\sum_{m=0}^{\infty} \frac{2 m+1}{2} P_{m}(\mu) e^{-\left[\sigma_{s 0}(E)-\sigma_{s m}(E)\right] \Delta s_{n}}\right\} d \mu \\
=\text { the probability that a particle, with polar } \\
\text { direction cosine } \mu=1 \text { at } s=s_{n}, \text { has a polar } \\
\text { direction cosine in } d \mu \text { about } \mu \text { at } s=s_{n+1} .
\end{gathered}
$$

Also, it is clear from symmetry considerations that

$$
\begin{gathered}
\frac{d \phi}{2 \pi}=\text { the probability that a particle, with polar direction cosine } \mu=1 \\
\text { at } s=s_{n}, \text { has an azimuthal angle in } d \phi \text { about } \phi \text { at } s=s_{n+1} .
\end{gathered}
$$

Eqs. (7) and (8) enable us to solve Eq. (3) by the following Monte Carlo simulation. We have assumed that at $s=s_{n}$, a particle's position, direction, and energy are $\underline{r}_{n}, \underline{\Omega}_{n}$, and $E_{n}$. Following the prescription given above and sampling from the probability distribution functions (7) and (8), we obtain $\mu_{n+1}$ and $\phi_{n+1}$, i.e., $\underline{\Omega}_{n+1}$. The remaining independent variables are unchanged. [See Eq. (6).] Hence,

$$
\begin{aligned}
& \underline{r}_{n+1}=\underline{r}_{n}, \\
& \underline{\Omega}_{n+1}: \text { Eqs. (7) and (8) } \\
& E_{n+1}=E_{n} .
\end{aligned}
$$


Finally, let us consider the transport process that includes slowing down but does not include space-dependence or angular scattering. This is described by Eq. (1) with $0=\underline{\Omega} \cdot \underline{\nabla} \psi=\sigma_{s}(E)=$ $\sigma_{s}\left(\underline{\Omega} \cdot \underline{\Omega^{\prime}}, E\right)$ :

$$
\frac{1}{v} \frac{\partial \psi}{\partial t}(\underline{r}, \underline{\Omega}, E, t)-\frac{\partial}{\partial E} \beta(E) \psi(\underline{r}, \underline{\Omega}, E, t)=0 .
$$

(We shall say that this describes "transport process C.") Eq. (9) is equivalent to the system:

$$
\begin{aligned}
& \frac{d t}{d s}=\frac{1}{v}, \\
& \frac{d E}{d s}=-\beta(E) ;, \\
& \frac{d}{d s} \beta(E) \psi(\underline{r}, \underline{\Omega}, E, t)=0 .
\end{aligned}
$$

Process $\mathrm{C}$, like process A, can be solved by a deterministic Monte Carlo procedure. If at $s=s_{n}$ a particle's position, direction, and energy are $\underline{r}_{n}, \underline{\Omega}_{n}$, and $E_{n}$, then Eqs. (10)-(12) imply that at $s=s_{n+1}$ the particle's position, direction, and energy are deterministically given by:

$$
\begin{aligned}
\underline{r}_{n+1} & =\underline{r}_{n}, \\
\underline{\Omega}_{n+1} & =\underline{\Omega}_{n}, \\
E_{n+1} & : \int_{E_{n+1}}^{E_{n}} \frac{d E}{\beta(E)}=\Delta s_{n} .
\end{aligned}
$$

In practice, the set $\left\{E_{n}, n \geq 0\right\}$ is precomputed and stored in a table.

We have seen that the transport processes A (with streaming but without angular scattering or slowing down), B (with angular scattering but without streaming or slowing down), and C (with slowing down but without streaming or angular scattering) can each be simulated by a relatively simple Monte Carlo method. However, the physical transport process described by Eq. (1) couples all these phenomena. To model this coupled process, the Condensed History algorithm alternates between the three simpler processes A, B, and C for "short" path length steps $\Delta s_{n}$.

Specifically, suppose that at $s=s_{n}$, the position, direction, and energy of a particle are given by $\underline{r}_{n}, \underline{\Omega}_{n}$, and $E_{n}$, and we wish to calculate the particle's position, direction, and energy at $s=s_{n+1}$. The basic Condensed History Algorithm, which we will hereafter call method 1a, consists of the following three steps:

1. Implement process A over $s_{n} \leq s \leq s_{n+1}$ to obtain

$$
\begin{aligned}
\underline{r}_{n+1 / 3} & =\underline{r}_{n}+\underline{\Omega}_{n} \Delta s_{n}, \\
\underline{\Omega}_{n+1 / 3} & =\underline{\Omega}_{n}, \\
E_{n+1 / 3} & =E_{n} .
\end{aligned}
$$

2. Next, implement process B over $s_{n} \leq s \leq s_{n+1}$, using $\underline{r}_{n+1 / 3}, \underline{\Omega}_{n+1 / 3}$, and $E_{n+1 / 3}$ as initial data, to obtain

$$
\begin{aligned}
& \underline{r}_{n+2 / 3}=\underline{r}_{n+1 / 3}, \\
& \underline{\Omega}_{n+2 / 3}: \text { Eqs. (7) and (8) } \\
& E_{n+2 / 3}=E_{n+1 / 3} .
\end{aligned}
$$


3. Finally, implement process C over $s_{n} \leq s \leq s_{n+1}$, using $\underline{r}_{n+2 / 3}, \underline{\Omega}_{n+2 / 3}$, and $E_{n+2 / 3}$ as initial data, to obtain

$$
\begin{aligned}
\underline{r}_{n+1} & =\underline{r}_{n+2 / 3} \\
\underline{\Omega}_{n+1} & =\Omega_{n+2 / 3} \\
E_{n+1} & : \int_{E_{n+1}}^{E_{n+2 / 3}} \frac{d E}{\beta(E)}=\Delta s_{n} .
\end{aligned}
$$

Thus, in step 1, the particle is moved a distance $\Delta s_{n}$, but its direction and energy are unchanged. In step 2, the particle's direction is altered but its position and energy are unchanged. Finally, in step 3, the particle's energy is altered, but its position and direction are unchanged. The geometry of this procedure is illustrated in Figure 1.

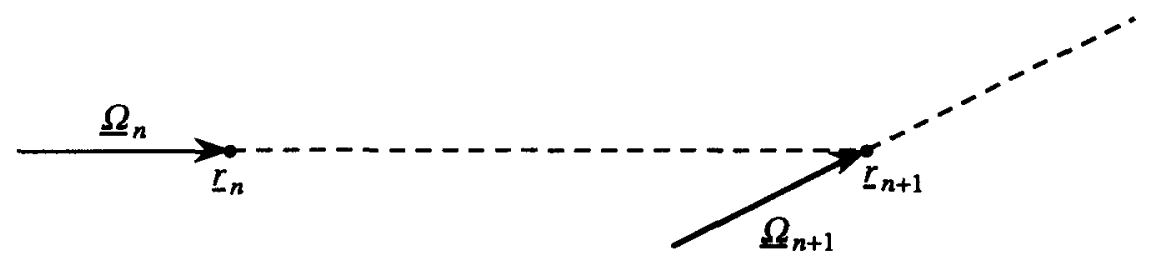

Figure 1: Method 1a

One hopes, of course, that the histories $\left\{\underline{r}_{n}, \underline{\Omega}_{n}, E_{n}, s_{n}\right.$ for $\left.n \geq 0\right\}$ provide an accurate statistical sampling of a true particle history at the discrete path-lengths $s_{n}$. However, as noted by Berger, ${ }^{3}$ one obvious discrepency between the physical and approximate process is that in each path-length step, Monte Carlo particles are constrained to lie on the ray determined by the initial direction and undergo no transverse displacements, whereas real electrons, which undergo many collisions in travelling a distance $\Delta s$, do undergo a transverse displacement. This is illustrated in Figure 2.

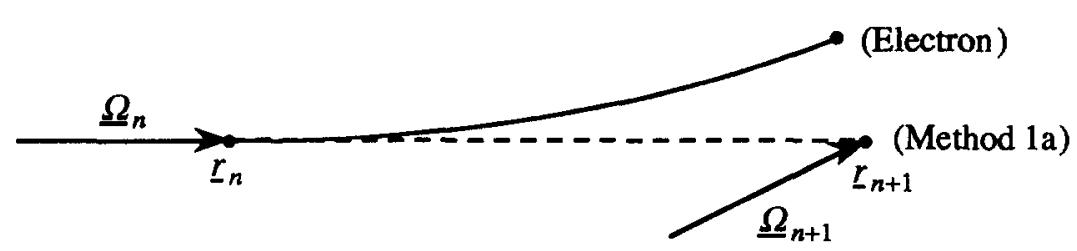

Figure 2: Transverse Displacements

One way to treat this difficulty is to interchange steps 1 and 2 ; thus, one first implements process $\mathrm{B}$, then $\mathrm{A}$, and then $\mathrm{C}$. Now the direction of the particle is changed prior to its spatial 
displacement, so there will be a transverse displacement. (We shall call this method $1 \mathrm{~b}$.) This is illustrated in Figure 3.

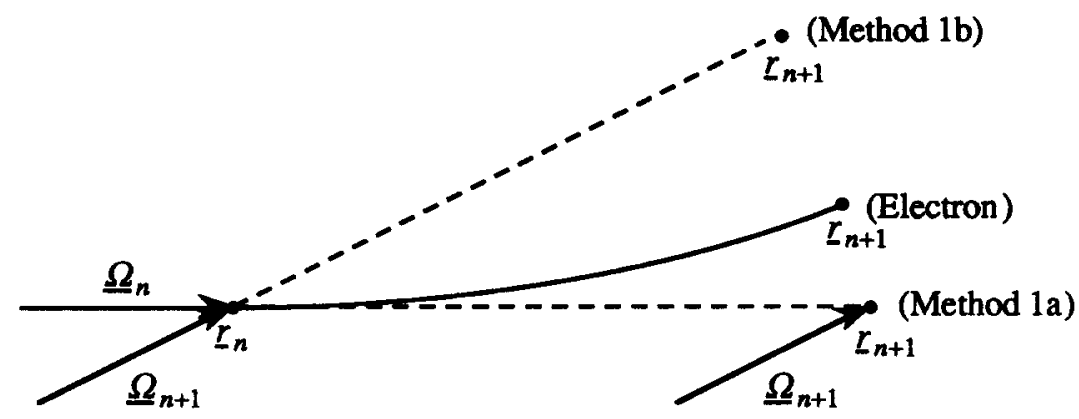

Figure 3: Methods 1a and 1b

However, the transverse displacement is now likely to be too large, because it is based on a direction of flight that is sampled from the Goudsmit-Saunderson distribution at the end of the path-length step, when it is more isotropic, rather than at a more representative value. [See Eq. (7).]

This difficulty can be treated by generalizing method $1 \mathrm{a}$ to the following method, discussed by Berger, ${ }^{3}$ which we shall call method 2:

1. Implement process A over $s_{n} \leq s \leq s_{n+1 / 2}=\left(s_{n}+s_{n+1}\right) / 2$ to obtain

$$
\begin{aligned}
\underline{r}_{n+1 / 4} & =\underline{r}_{n}+\underline{\Omega}_{n} \frac{\Delta s_{n}}{2}, \\
\Omega_{n+1 / 4} & =\underline{\Omega}_{n}, \\
E_{n+1 / 4} & =E_{n} .
\end{aligned}
$$

2. Next, implement process B over $s_{n} \leq s \leq s_{n+1}$, using $\underline{r}_{n+1 / 4}, \underline{\Omega}_{n+1 / 4}$, and $E_{n+1 / 4}$ as initial data, to obtain

$$
\begin{aligned}
& \underline{r}_{n+2 / 4}=\underline{r}_{n+1 / 4}, \\
& \Omega_{n+2 / 4}: \text { Eqs. (7) and (8), } \\
& E_{n+2 / 4}=E_{n+1 / 4} .
\end{aligned}
$$

3. Next, implement process $C$ over $s_{n} \leq s \leq s_{n+1}$, using $\underline{r}_{n+2 / 4}, \underline{\Omega}_{n+2 / 4}$, and $E_{n+2 / 4}$ as initial data, to obtain

$$
\begin{aligned}
\underline{r}_{n+3 / 4} & =\underline{r}_{n+2 / 4}, \\
\underline{\Omega}_{n+3 / 4} & =\underline{\Omega}_{n+2 / 4}, \\
E_{n+3 / 4} & : \int_{E_{n+3 / 4}}^{E_{n}+2 / 4} \frac{d E}{\beta(E)}=\Delta s_{n} .
\end{aligned}
$$


4. Finally, implement process A over $s_{n+1 / 2} \leq s \leq s_{n+1}$, using $\underline{r}_{n+3 / 4}, \underline{\Omega}_{n+3 / 4}$, and $E_{n+3 / 4}$ as initial data, to obtain

$$
\begin{aligned}
\underline{r}_{n+1} & =\underline{r}_{n+3 / 4}+\underline{\Omega}_{n+3 / 4} \frac{\Delta s_{n}}{2} \\
\underline{\Omega}_{n+1} & =\underline{\Omega}_{n+3 / 4} \\
E_{n+1} & =E_{n+3 / 4}
\end{aligned}
$$

Now, the modification of the particle's direction conceptually occurs midway in the path-length step, so one is likely to obtain a more accurate transverse displacement (see Figure 4).

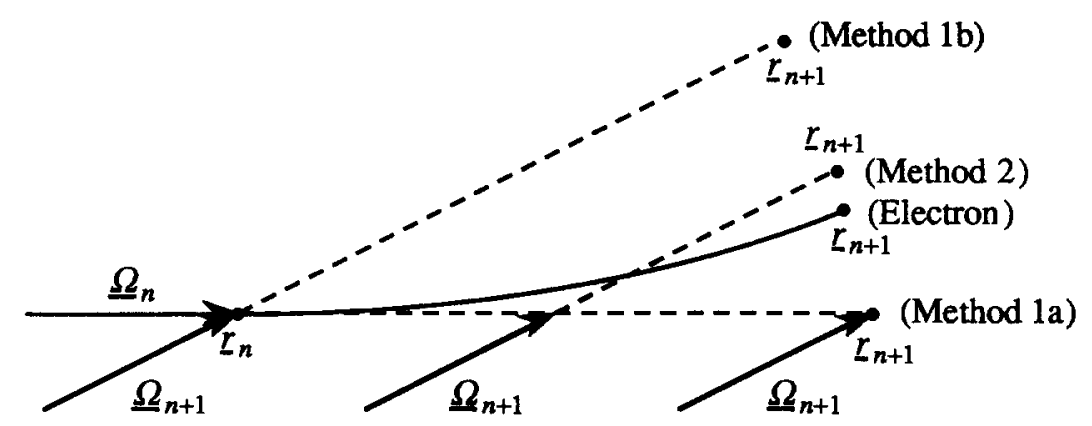

Figure 4: Methods 1a, 1b, and 2

We note that steps 3 and 4 in this method can be interchanged with no change in the final values of $\underline{r}_{n+1}, \Omega_{n+1}$, and $E_{n+1}$. We also note that in all of the methods that we have discussed thus far, the Goudsmit-Saunderson distribution is evaluated at $E_{n}$. If we implement process $C$ before process $B$ in all of the above methods, the Goudsmit-Saunderson distribution will be evaluated at $E_{n+1}$. Either way, a value of energy is used that is less representative during the path-length step than some intermediate value.

This conceptual difficulty can be treated by generalizing method 2 to the following method, which has not been proposed previously, and which we shall call method 3:

1. Implement process A over $s_{n} \leq s \leq s_{n+1 / 2}$ to obtain

$$
\begin{aligned}
\underline{r}_{n+1 / 5} & =\underline{r}_{n}+\underline{\Omega}_{n} \frac{\Delta s_{n}}{2}, \\
\underline{\Omega}_{n+1 / 5} & =\underline{\Omega}_{n}, \\
E_{n+1 / 5} & =E_{n} .
\end{aligned}
$$

2. Next, implement process C over $s_{n} \leq s \leq s_{n+1 / 2}$, using $\underline{r}_{n+1 / 5}, \underline{\Omega}_{n+1 / 5}$, and $E_{n+1 / 5}$ as initial data, to obtain 


$$
\begin{aligned}
& \underline{r}_{n+2 / 5}=\underline{r}_{n+1 / 5}, \\
& \underline{\Omega}_{n+2 / 5}=\underline{\Omega}_{n+1 / 5}, \\
& E_{n+2 / 5}: \int_{E_{n+2 / 5}}^{E_{n+1 / 5}} \frac{d E}{\beta(E)}=\frac{\Delta s_{n}}{2} .
\end{aligned}
$$

3. Next, implement process B over $s_{n} \leq s \leq s_{n+1}$, using $\underline{r}_{n+2 / 5}, \underline{\Omega}_{n+2 / 5}$, and $E_{n+2 / 5}$ as initial data, to obtain

$$
\begin{aligned}
& \underline{r}_{n+3 / 5}=\underline{r}_{n+2 / 5}, \\
& \underline{\Omega}_{n+3 / 5}: \text { Eqs. (7) and (8) }, \\
& E_{n+3 / 5}=E_{n+2 / 5} .
\end{aligned}
$$

4. Next, implement process $C$ over $s_{n+1 / 2} \leq s \leq s_{n+1}$, using $\underline{r}_{n+3 / 5}, \underline{\Omega}_{n+3 / 5}$, and $E_{n+3 / 5}$ as initial data, to obtain

$$
\begin{aligned}
\underline{r}_{n+4 / 5} & =\underline{r}_{n+3 / 5}, \\
\underline{\Omega}_{n+4 / 5} & =\underline{\Omega}_{n+3 / 5}, \\
E_{n+4 / 5} & : \int_{E_{n+4 / 5}}^{E_{n+3 / 5}} \frac{d E}{\beta(E)}=\frac{\Delta s_{n}}{2} .
\end{aligned}
$$

5. Finally, implement process A over $s_{n+1 / 2} \leq s \leq s_{n+1}$, using $\underline{r}_{n+4 / 5}, \underline{\Omega}_{n+4 / 5}$, and $E_{n+4 / 5}$ as initial data, to obtain

$$
\begin{aligned}
\underline{r}_{n+1} & =\underline{r}_{n+4 / 5}+\underline{\Omega}_{n+1} \frac{\Delta s_{n}}{2}, \\
\underline{\Omega}_{n+1} & =\underline{\Omega}_{n+4 / 5}, \\
E_{n+1} & =E_{n+4 / 5} .
\end{aligned}
$$

Now, the Goudsmit-Saunderson distribution is evaluated at an intermediate energy $\left(E_{n+2 / 5}\right)$, rather than at the beginning or final energy in the path-length step. This should yield a more accurate method. Also, the extra computational work required to implement method 3 is trivial.

In this section we have used physical arguments to derive the basic Condensed History Algorithm (method 1a), a simple variation (method 1b), and two somewhat more complicated variations (methods 2 and 3 ). Methods $1 \mathrm{a}$ and 2 have been described by Berger; ${ }^{3}$ method 3 is new. In the next section, we show that for $O(1)$ values of $s$, methods $1 \mathrm{a}, 1 \mathrm{~b}$, and 2 have an $O(\Delta s)$ truncation error, while method 3 has an $O\left(\Delta s^{2}\right)$ truncation error.

Of course, in practical Monte Carlo simulations, there are additional statistical errors due to the use of a finite number of Monte Carlo particles. However, in this paper we are concerned with the magnitude of the truncation errors that are inherent in each version of the Condensed History Algorithm; we are not concerned with the statistical errors, which depend on the number of Monte Carlo particles used in a calculation. 


\section{MATHEMATICAL DERIVATION}

Let us define the "streaming" operator $A$ as

$$
A \psi(\underline{r}, \underline{\Omega}, E, s)=\underline{\Omega} \cdot \underline{\nabla} \psi(\underline{r}, \underline{\Omega}, E, s),
$$

the "angular scattering" operator $B$ as

$$
B \psi(\underline{r}, \underline{\Omega}, E, s)=\sigma_{s}(E) \psi(\underline{r}, \underline{\Omega}, E, s)-\int \sigma_{s}\left(\underline{\Omega} \cdot \underline{\Omega^{\prime}}, E\right) \psi\left(\underline{r}, \underline{\Omega^{\prime}}, E, s\right) d \Omega^{\prime},
$$

and the "continuous slowing-down" operator $C$ as

$$
C \psi(\underline{r}, \underline{\Omega}, E, s)=-\frac{\partial}{\partial E} \beta(E) \psi(\underline{r}, \underline{\Omega}, E, s) .
$$

Then, Eq. (1) can be written

$$
\frac{\partial}{\partial s} \psi(\underline{r}, \underline{\Omega}, E, s)+(A+B+C) \psi(\underline{r}, \underline{\Omega}, E, s)=0 .
$$

If we assume that $\psi\left(\underline{r}, \underline{\Omega}, E, s_{n}\right)$ is known, then the solution of Eq. (22) with this given initial condition is

$$
\psi(\underline{r}, \underline{\Omega}, E, s)=e^{-(A+B+C)\left(s-s_{n}\right)} \psi\left(\underline{r}, \underline{\Omega}, E, s_{n}\right) \quad, \quad s \geq s_{n}
$$

Hence, the solution at $s=s_{n+1}$ is

$$
\psi\left(\underline{r}, \underline{\Omega}, E, s_{n+1}\right)=e^{-(A+B+C) \Delta s_{n}} \psi\left(\underline{r}, \underline{\Omega}, E, s_{n}\right) .
$$

[Note: the operator

$$
e^{-(A+B+C)\left(s-s_{n}\right)} \equiv U\left(s-s_{n}\right)
$$

is a $\mathrm{C}_{0}$-semigroup with infinitesimal generator $A+B+C$. The notation and concepts behind the semigroup operator are thoroughly discussed for the transport equation in the monograph by Greenberg, van der Mee, and Protopopescu. ${ }^{6}$ ]

Now we shall compare the exact solution [Eq. (23)] of Eq. (22) to the approximate solutions obtained by the various operator-split methods discussed in the previous section. We note that for a bounded operator $L$, the expansion

$$
e^{-L \Delta s}=\sum_{n=0}^{\infty} \frac{(-\Delta s)^{n}}{n !} L^{n}
$$

is valid for all $\Delta s$, whereas for an unbounded operator,

$$
e^{-L \Delta s} \psi=\left(1-\Delta s L+\frac{\Delta s^{2}}{2} L^{2}\right) \psi+O\left(\Delta s^{3}\right)
$$

is valid if $\psi$ is in the domain of $L^{3}$. If $L=A+B+C$ is the transport operator, whose unbounded parts are the differential streaming and slowing down operators, then the domain of $L^{3}$ is the set of functions $\psi(\underline{r}, \underline{\Omega}, E, s)$ that are sufficiently smooth functions of $\underline{r}$ and $E$. In this paper we will work with truncated expansions of the form (24), and we will tacitly assume that the initial data 
is sufficiently smooth to justify these expansions. Now, method 1a, defined by Eqs. (13)-(21), can be written as:

$$
\begin{aligned}
\frac{\partial \tilde{\psi}}{\partial s}(\underline{r}, \underline{\Omega}, E, s)+A \tilde{\psi}(\underline{r}, \underline{\Omega}, E, s) & =0, \quad s_{n} \leq s \leq s_{n+1} \\
\tilde{\psi}\left(\underline{r}, \underline{\Omega}, E, s_{n}\right) & =\psi\left(\underline{r}, \underline{\Omega}, E, s_{n}\right) ; \\
\frac{\partial \hat{\psi}}{\partial s}(\underline{r}, \underline{\Omega}, E, s)+B \hat{\psi}(\underline{r}, \underline{\Omega}, E, s) & =0, \quad s_{n} \leq s \leq s_{n+1} \\
\hat{\psi}\left(\underline{r}, \underline{\Omega}, E, s_{n}\right) & =\tilde{\psi}\left(\underline{r}, \underline{\Omega}, E, s_{n+1}\right) ; \\
\frac{\partial \psi}{\partial s}(\underline{r}, \underline{\Omega}, E, s)+C \psi(\underline{r}, \underline{\Omega}, E, s) & =0, \quad s_{n} \leq s \leq s_{n+1} \\
\psi\left(\underline{r}, \underline{\Omega}, E, s_{n}\right) & =\hat{\psi}\left(\underline{r}, \underline{\Omega}, E, s_{n+1}\right) .
\end{aligned}
$$

More precisely, Eqs. (13)-(21) describe a Monte Carlo simulation of Eqs. (25)-(30). Eqs. (25) and (26) imply

$$
\tilde{\psi}\left(\underline{r}, \underline{\Omega}, E, s_{n+1}\right)=e^{-A \Delta s_{n}} \psi\left(\underline{r}, \underline{\Omega}, E, s_{n}\right) \quad ;
$$

Eqs. (27) and (28) imply

$$
\hat{\psi}\left(\underline{r}, \underline{\Omega}, E, s_{n+1}\right)=e^{-B \Delta s_{n}} \tilde{\psi}\left(\underline{r}, \underline{\Omega}, E, s_{n+1}\right) \quad ;
$$

and Eqs. (29) and (30) imply

$$
\psi\left(\underline{r}, \underline{\Omega}, E, s_{n+1}\right)=e^{-C \Delta s_{n}} \hat{\psi}\left(\underline{r}, \underline{\Omega}, E, s_{n+1}\right) .
$$

Combining these three equations, we obtain (for method 1a)

$$
\psi\left(\underline{r}, \underline{\Omega}, E, s_{n+1}\right)=e^{-C \Delta s_{n}} e^{-B \Delta s_{n}} e^{-A \Delta s_{n}} \psi\left(\underline{r}, \underline{\Omega}, E, s_{n}\right)
$$

Now, we have

$$
e^{-(A+B+C) \Delta s} \psi=\left[1-\Delta s(A+B+C)+\frac{\Delta s^{2}}{2}(A+B+C)^{2}\right] \psi+O\left(\Delta s^{3}\right)
$$

Noting that $B$ commutes neither with $A$ nor $C$, we obtain

$$
\begin{gathered}
e^{-C \Delta s} e^{-B \Delta s} e^{-A \Delta s} \psi \\
=\left(1-\Delta s C+\frac{\Delta s^{2}}{2} C^{2}\right)\left(1-\Delta s B+\frac{\Delta s^{2}}{2} B^{2}\right)\left(1-\Delta s A+\frac{\Delta s^{2}}{2} A^{2}\right) \psi+O\left(\Delta s^{3}\right) \\
=\left[1-\Delta s(A+B+C)+\frac{\Delta s^{2}}{2}\left(A^{2}+B^{2}+C^{2}+2 B A+2 C A+2 C B\right)\right] \psi+O\left(\Delta s^{3}\right) \\
=e^{-(A+B+C) \Delta s} \psi+O\left(\Delta s^{2}\right) .
\end{gathered}
$$

Thus, method 1a creates an $O\left(\Delta s^{2}\right)$ error within each path-length step. After $O(1 / \Delta s)$ path-length steps [i.e., $s=O(1)$ ] the error is $O(\Delta s)$.

The analysis of method $1 \mathrm{~b}$ is identical to that of method 1a, except that the operators $A$ and $B$ are interchanged. Thus, method $1 \mathrm{~b}$ also has an $O(\Delta s)$ error. 
The operator that defines method 2 is easily seen to be

$$
\begin{gathered}
e^{-\frac{1}{2} A \Delta s} e^{-C \Delta s} e^{-B \Delta s} e^{-\frac{1}{2} A \Delta s} \psi \\
=\left\{1-\Delta s(A+B+C)+\frac{\Delta s^{2}}{2}\left[(A+B+C)^{2}+C B-B C\right]\right\} \psi+O\left(\Delta s^{3}\right) \\
=e^{-(A+B+C) \Delta s} \psi+O\left(\Delta s^{2}\right) .
\end{gathered}
$$

Thus for $O(1)$ values of $s$, method 2 has an $O(\Delta s)$ error unless the operators $B$ and $C$ commute. (This happens only if the angular scatering cross sections are independent of $E$.)

Finally, the operator that defines method 3 is

$$
e^{-\frac{1}{2} A \Delta s} e^{-\frac{1}{2} C \Delta s} e^{-B \Delta s} e^{-\frac{1}{2} C \Delta s} e^{-\frac{1}{2} A \Delta s} \psi=e^{-(A+B+C) \Delta s} \psi+O\left(\Delta s^{3}\right)
$$

We note that since $A$ and $C$ commute,

$$
e^{-\frac{1}{2} A \Delta s} e^{-\frac{1}{2} C \Delta s}=e^{-\frac{1}{2} C \Delta s} e^{-\frac{1}{2} A \Delta s}=e^{-\frac{1}{2}(A+C) \Delta s}
$$

so method 3 is also defined by

$$
e^{-\frac{1}{2}(A+C) \Delta s} e^{-B \Delta s} e^{-\frac{1}{2}(A+C) \Delta s} \psi=e^{-(A+B+C) \Delta s} \psi+O\left(\Delta s^{3}\right)
$$

Either way, we see that for $O(1)$ values of $s$, method 3 has an $O\left(\Delta s^{2}\right)$ error.

We conclude that versions 1a, 1b, 2, and 3 of the Condensed History Algorithm are Monte Carlo simulations of different operator-split strategies for solving Eq. (1). These operator-split strategies have different orders of truncation error in the path-length step $\Delta s$. In numerical simulations, these different truncation errors will be observed by running the Monte Carlo codes with enough histories that the statistical errors are sufficiently small.

\section{DISCUSSION}

In this paper, we have justified several versions of the Condensed History Algorithm as Monte Carlo simulations of different operator-split strategies for electron transport problems. Because the Monte Carlo simulations of the streaming process, the angular scattering process, and the continuous slowing down process are exact, then the accuracies of the various versions of the Condensed History Algorithm depend only on the accuracies of the underlying operator split strategies, given a sufficient number of Monte Carlo particles that the statistical errors can be ignored.

In particular, we have shown that previously-proposed versions of the method have $O(\Delta s)$ errors, but that by subdividing a path-length step $s_{n} \leq s \leq s_{n+1}$ into two substeps and implementing some of the processes over substeps (and in a certain order), one can obtain a new method whose errors are $O\left(\Delta s^{2}\right)$. There is no doubt that by using still smaller substeps, one can devise more complex operator split strategies that are $O\left(\Delta s^{3}\right)$. However, we will not pursue this here.

An alternate way to develop more accurate strategies is to "mix" the order in which the processes $\mathrm{A}, \mathrm{B}$, and $\mathrm{C}$ are implemented in each path-length step. For example, we note that

$$
\left(e^{-A \Delta s} e^{-C \Delta s} e^{-B \Delta s}\right)\left(e^{-B \Delta s} e^{-C \Delta s} e^{-A \Delta s}\right)=e^{-(A+B+C) 2 \Delta s}+O\left(\Delta s^{3}\right)
$$

The operator-split defined on the left side of this equation spans two path-length steps; it can be interpreted as a variation of method 1a (let us call it method 1c) implemented for the odd-numbered 
steps, and a second variation of method 1a in which processes A and B are interchanged (let us call this method 1d) for the even-numbered steps. Then, although methods $1 \mathrm{c}$ and $1 \mathrm{~d}$ individually have $O(\Delta s)$ errors, the composite method described above has $O\left(\Delta s^{2}\right)$ errors at the end of every second path-length step. It is easy to see that this new method is really a version of method 2 that spans two path-length steps rather than one. Nevertheless, this concept of "mixing" the order in which processes A, B, and C are implemented can clearly be generalized to yield methods that have higher order truncation errors. We shall not consider this further here.

Another possible extension of this work would involve applying the Monte Carlo operator-split strategy to more realistic transport models. For example, Eq. (1) is an approximation to a more general transport equation

$$
\frac{1}{v} \frac{\partial \psi}{\partial t}(\underline{r}, \underline{\Omega}, E, t)+(A+D) \psi(\underline{r}, \underline{\Omega}, E, t)=0,
$$

where $A$ is again the streaming operator

$$
A \psi(\underline{r}, \underline{\Omega}, E, t)=\underline{\Omega} \cdot \underline{\nabla} \psi(\underline{r}, \underline{\Omega}, E, t)
$$

and $D$ is the angular and energy scattering operator

$$
D \psi(\underline{r}, \underline{\Omega}, E, t)=\sigma_{s}(E) \psi(\underline{r}, \underline{\Omega}, E, t)-\iint \sigma_{s}\left(\underline{\Omega} \cdot \underline{\Omega^{\prime}}, E^{\prime} \rightarrow E\right) \psi\left(\underline{r}, \underline{\Omega^{\prime}}, E^{\prime}, t\right) d \Omega^{\prime} d E
$$

It is known that the conventional Condensed History Algorithm becomes inaccurate for low electron energies, where the continuous slowing down approximation becomes poor. ${ }^{7-9}$ By basing a Monte Carlo scheme on a more accurate physical model, it may be possible to recover much of the lost accuracy. However, using a more complex scattering operator such as $D$ may lead to considerable difficulty in developing workable probability distribution functions for the Monte Carlo simulation of the angular and energy scattering process. Again, the detailed consideration of such a scheme must await future work.

\section{ACKNOWLEDGEMENTS}

I would like to thank Jim Morel, Clinton Ballanger, and James Rathkopf for several helpful discussions on the Condensed History Algorithm.

\section{REFERENCES}

1. Lewis H.W. (1950) Multiple scattering in an infinite medium. Phys. Rev. 78, 526.

2. Hebbard D.F. and Wilson P.R. (1955) The effect of multiple scattering on electron energy loss distributions. Australian J. Phys. 8, 90.

3. Berger M.J. (1963) Monte Carlo calculation of the penetration and diffusion of fast charged particles. In Methods in Computational Physics, Volume 1, Alder B., Fernbach S., and Rotenburg M., eds., Academic Press, New York, 135-215.

4. Case K.M. and Zweifel P.F. (1967) Linear Transport Theory, Addison-Wesley, Reading, Mass.

5. Goudsmit S. and Saunderson J.L. (1940) Multiple scattering of electrons. Phys. Rev. 57, 24. 
6. Greenberg W., van der Mer C., and Protopopescu V. (1987) Boundary Value Problems in Abstract Kinetic Theory, Birkhäuser Verlag, Basel.

7. Ballinger C.T. (1991) The response history Monte Carlo hethod for electron transport. Ph.D. Thesis, Dept. of Nuclear Engineering, University of Michigan.

8. Ballinger C.T., Cullen D.E., Perkins S.T., Rathkopf J.A., Martin W.R., and Wilderman S.J. (1991) Single-scatter Monte Carlo compared to condensed history for low energy electrons. Lawrence Livermore National Laboratory Report UCRL-JC-107067; to appear in Nucl. Inst. and Methods in Phys. Research, Sec. B.

9. Ballinger C.T., Rathkopf J.A., and Martin W.R. (1991) Limitations of the condensed history method for low-energy electrons. Trans. Am. Nucl. Soc. 64, 306. 